\title{
Angiopoietin-like proteins inhibitors: New horizons in the treatment of atherogenic dyslipidemia and familial hypercholesterolemia
}

\author{
Stanisław Surma ${ }^{1}$, Monika Romańczyk ${ }^{1}$, Krzysztof J. Filipiak $^{2}$ \\ ${ }^{1}$ Faculty of Medical Sciences in Katowice, Medical University of Silesia, Katowice, Poland \\ ${ }^{2} 1^{\text {st }}$ Department of Cardiology, Medical University of Warsaw, Poland
}

\begin{abstract}
Angiopoietin-like proteins (ANGPTL) are involved in the regulation of numerous physiological and biochemical processes. ANGPTL3, 4 and 8, which are involved in the regulation of lipoprotein metabolism, are particularly important. ANGPTL3, 4 and 8 have been shown to regulate triglyceride availability depending on the nutritional status of the body. In addition, a deficiency of these proteins has been found to cause hypolipidemia (reduction of all lipid fractions). Increases in ANGPTL3, 4 and 8 appear to be associated with cardiovascular risk. Animal studies indicate that the use of ANGPTL3 (evinacumab) inhibitors significantly reduces plasma total cholesterol, triglycerides and low-density lipoprotein concentrations. The use of evinacumab in clinical trials also led to the normalization of plasma lipid concentrations in patients with atherogenic dyslipidemia and homozygous familial hypercholesterolemia. The results of these studies indicate that evinacumab may in the future be used in the treatment of lipid disorders, especially those with hypertriglyceridemia. (Cardiol J 2023; 30, 1: 131-142)

Key words: angiopoietin-like proteins (ANGPTL), evinavumab, ANGPTL3- $\mathrm{L}_{\mathrm{Rx}}$, familial hypercholesterolemia
\end{abstract}

Angiopoietin-like proteins: Structure, function of lipid metabolism regulation

Interest of angiopoietin-like proteins (ANGPTL) in the context of lipid disorders treatment, especially in the treatment of hypertriglyceridemia, results from their multidirectional influence on the regulation of the lipid metabolism [1].

So far, 8 proteins belonging to the family of ANGPTL1-ANGPTL8 have been discribed. ANGPTL are members of the vascular endothelial growth factor (VEGF) family. All ANGPTLs have a similar structure. The exception is ANGPTL8 which does not have a fibrinogen-like domain at the carboxy terminus [2]. These proteins are involved in stem cell expansion, inflammation regulation, tissue remodeling and angiogenesis [3, 4]. ANGPTL3,
ANGPTL4 and ANGPTL8 are primarily involved in lipid metabolism (Fig. 1) [1,5].

ANGPTL3 is a glycoprotein synthesized mainly by the liver and kidneys. Biological activation of ANGPTL3 occurs intra- and extracellularly. About $50 \%$ of the ANGPTL3 precursor form produced is proteolytically cleaved in the cell with furin (this enzyme also participates in the activation of natriuretic peptides) to a form with greater biological activity. The remaining $50 \%$ of the precursor form ANGPTL3 is secreted into the extracellular space, where it may undergo proteolytic cleavage by furin or proprotein convertase subtilisin/kexin type 6 (PCSK6). As a result of the action of furin or PCSK6, the N-terminal domain capable of inhibiting lipoprotein lipase (LPL) activity is exposed and its activity is greater than in the precursor

Address for correspondence: Prof. Krzysztof J. Filipiak, $1^{\text {st }}$ Department of Cardiology, Medical University of Warsaw, ul. Banacha 1a, block D, 02-097 Warszawa, Poland, e-mail: krzysztof.filipiak@wum.edu.pl

Received: 18.06.2020 Accepted: 25.09.2020 Early publication date: 14.01.2021

This article is available in open access under Creative Common Attribution-Non-Commercial-No Derivatives 4.0 International (CC BY-NC-ND 4.0) license, allowing to download articles and share them with others as long as they credit the authors and the publisher, but without permission to change them in any way or use them commercially. 


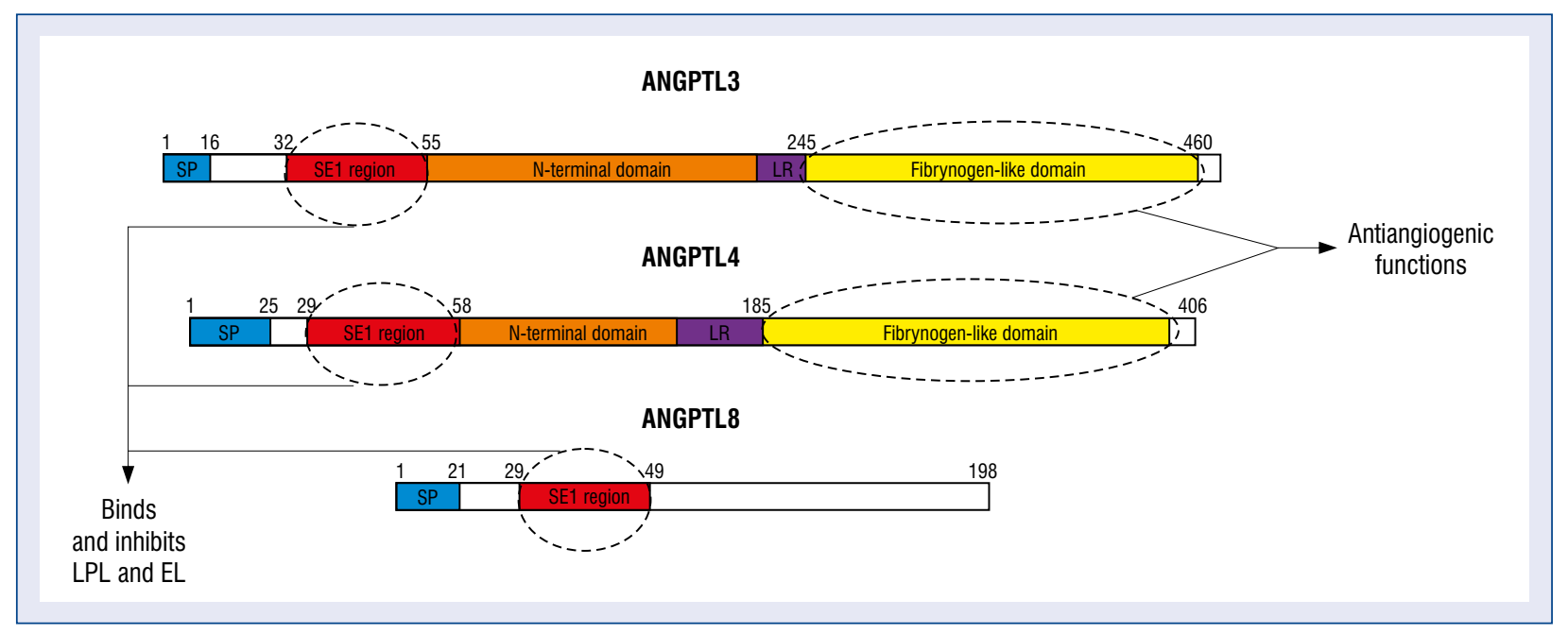

Figure 1. Schematic representation of the structure of angiopoietin-like proteins 3, 4, and 8 (ANGPTL3, ANGPTL4, and ANGPTL8). Based on [1,5]; EL — endothelial lipase; LPL — lipoprotein lipase; LR — linker region; SE1 region — specific epitope 1.

form ANGPTL3 [6]. ANGPTL3 circulates in both forms in the plasma [6]. In addition, it is known that other enzymes, such as PCSK2, PCSK4, PCSK5 and PCSK7, can also catalyze the proteolytic cleavage of the ANGPTL3 precursor form [3]. Interestingly, it has been shown that ANGPTL3 cleavage appears to be facilitated by ANGPTL8 (lipasin). ANGPTL8 is secreted by the liver into the circulation, where it interacts with ANGPTL3 for cleavage and forms a complex with the N-terminal fragment of ANGPTL3. The complex, like the free N-terminal fragment of ANGPTL3 [7]. Formation of the ANGPTL8-ANGPTL3 complex induces structural changes in ANGPTL8 that reveal a motif that inhibits LPL inhibition. Thus, ANGPTL8 remains inactive until it forms a complex with ANGPTL3. Furthermore, the ability of the ANGPTL8/ANGPTL3 complex to inhibit LPL is known to depend on the active LPL inhibitory motif in ANGPTL8 [8]. This is confirmed by the observations that LPL inhibition by the ANGPTL3/ /ANGPTL8 complex could not be reversed by the anti-ANGPTL3 blocking antibody [8]. The factors regulating ANGPTL3 transcription are LXR (liver $\mathrm{X}$ receptors) and nuclear factor 1 hepatocyte alpha $(\mathrm{HNF}-1 \alpha)$ [9]. In an interesting study by Foka et al. [10] showed that ANGPTL3 is negatively regulated by hepatitis $\mathrm{C}$ virus (HCV) in vivo and in vitro. The HCV core suppresses ANGPTL3 expression by loss of HNF- $1 \alpha$ binding activity and blocking LXR/RXR (retinoid X receptor) transactivation. The presumed resulting increase in serum lipid clearance and uptake by the liver may support HCV replication and persistence [10].

ANGPTL4 is a glycoprotein synthesized by many tissues, including adipose tissue, liver, intestines and muscles [11]. The expression of the ANGPTL4 gene is regulated by hunger and satiety. In particular, starvation enhances ANGPTL4 expression [1]. In addition, ANGPTL4 gene expression is also stimulated by receptor ligands activated by peroxisome proliferators (PPAR- $\alpha$, $-\delta$ and $\gamma)$ [11].

It is known that ANGPTL3, ANGPTL4 and ANGPTL8, like C-III apolipoprotein, reduce LPL activity to varying degrees [1]. The basic function of LPL is the hydrolysis of triacylglycerols (TG) transported in chylomicrons and very low-density lipoproteins (VLDL). As a result, residual chylomicrons (so-called chylomicron remnants) and intermediate density lipoproteins (IDL), which are precursors of low-density lipoproteins (LDL), are formed (Fig. 2) [12, 13].

ANGPTL3 reduces plasma VLDL and chylomicrons concentrations by reducing LPL activity and promoting lipolysis in adipose tissue. Furthermore, ANGPTL3 has been shown to reduce endothelial lipase (EL) activity [14]. This enzyme performs similar functions to LPL. It is known that EL is synthesized by endothelial cells and has antiatherosclerotic properties. EL catalyzes the hydrolysis reaction of phospholipids contained in high-density lipoproteins (HDLs), which increases the rate of HDL circulation, 


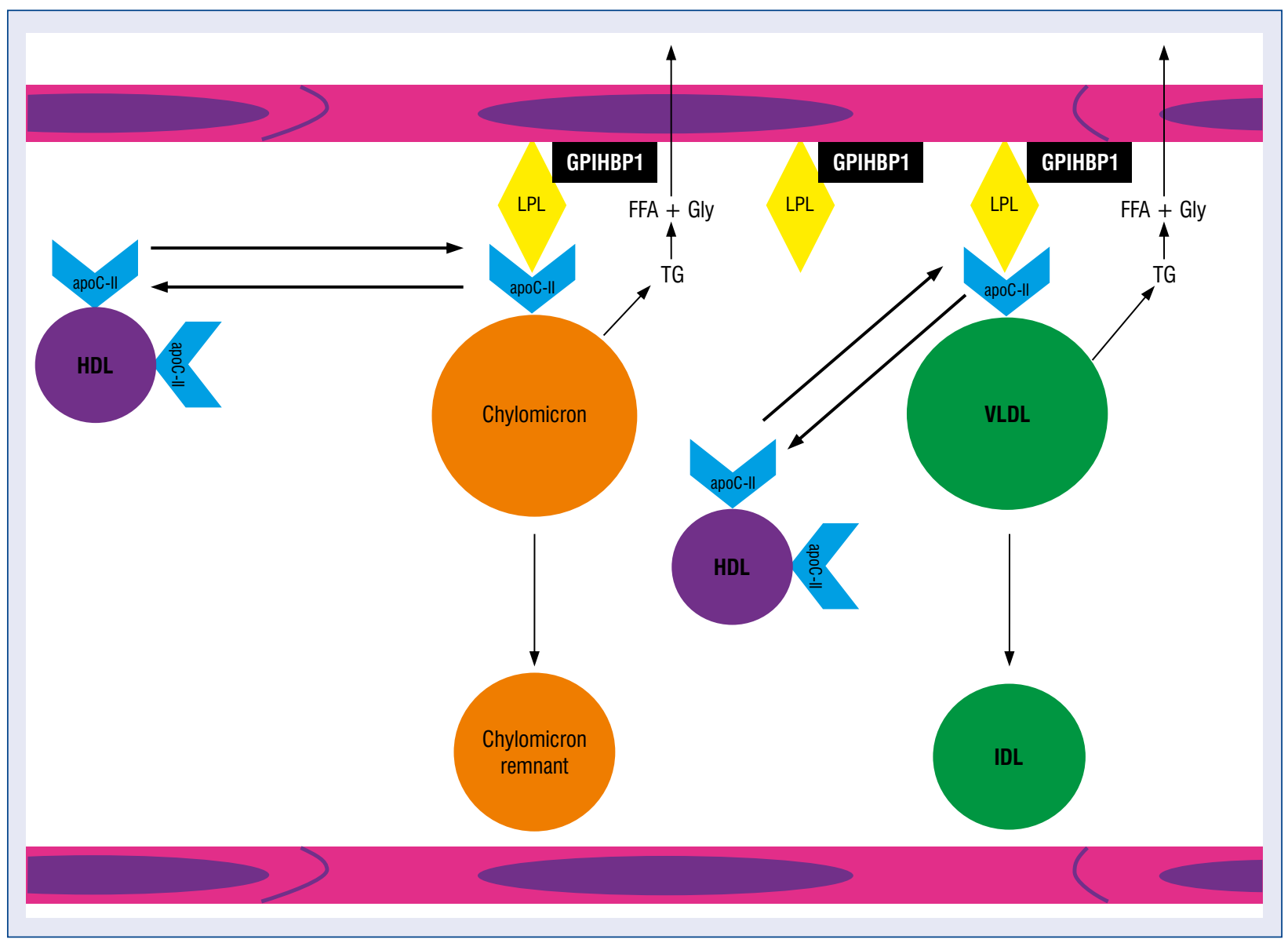

Figure 2. Participation lipoprotein lipase (LPL) in lipoprotein metabolism. Based on [13]; apoC-II - apolipoprotein C-II; FFA — free fatty acids; Gly — glycerol; GPIHBP1 - glycosylphosphatidylinositol anchored high density lipoprotein binding protein 1; HDL — high-density lipoprotein; IDL — intermediate-density lipoprotein; TG — triacylglycerols; VLDL - very low-density lipoprotein.

which leads to a decrease in plasma cholesterol concentration [15]. To date, however, the mechanism by which ANGPTL3 reduces LPL activity is not fully understood [1]. As indicated by Liu et al. [16] ANGPTL3 may increase LPL cleavage by endogenous furin and PACE4, but not by PCSK5. This mechanism is specific for LPL, but not for EL. Moreover, ANGPTL3 enhances LPL cleavage in the presence of either heparan sulfate proteoglycans or glycosylphosphatidylinositol-anchored high-density lipoprotein-binding protein 1 (GPIHBP1). By enhancing LPL cleavage, ANGPTL3 dissociates LPL from the cell surface, inhibiting both the catalytic and noncatalytic functions of LPL [16]. Another potential mechanism of action of ANGPTL3 is to promote TG accumulation in adipocytes [17]. Chi et al. [18] found that ANGPTL4 by attaching to LPL reduces its affinity for GPIHBP1. The biological function of ANGPTL8 is dependent on ANGPTL3 and also consists in reducing LPL stimulation [19].

Kovrov et al. [20] showed that ANGPTL3, ANGPTL4 and ANGPTL8 reduce LPL activity by causing its cleavage from dimeric (biologically active) to monomeric (biologically inactive) [20, 21].

The main physiological role of ANGPTL3, ANGPTL4 and ANGPTL8 resulting from the reduction of LPL excitation is the regulation of TG distribution (Fig. 3) [22].

During fed, ANGPTL3 and ANGPTL8 promote TG deposition in white adipose tissue by specifically inhibiting LPL activity in oxidative tissues (heart, skeletal muscle and brown adipose tissue). During exercise, starvation, and exposure to low temperatures, ANGPTL4 inhibits local LPL activity in white adipose tissue to provide sufficient energy to the heart, skeletal muscles, and brown adipose tissue (Fig. 3) [1, 23]. ANGPTL8 sup- 


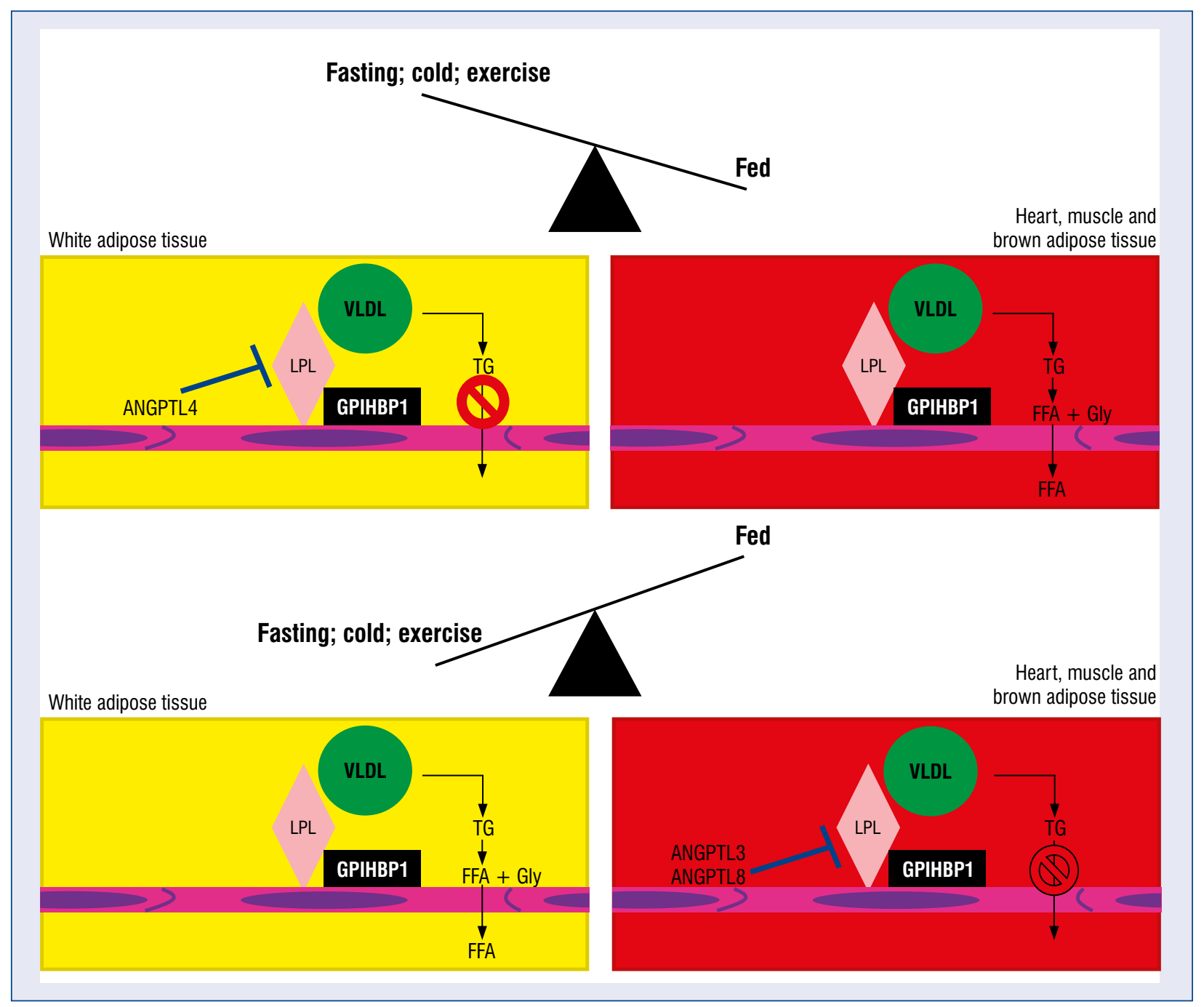

Figure 3. Lipid partitioning by angiopoietin-like proteins (ANGPTL). Based on [23]; FFA — free fatty acids; Gly — glicerol; GPIHBP1 — glycosylphosphatidylinositol anchored high density lipoprotein binding protein 1; LPL — lipoprotein lipase; TG — triacylglycerols; VLDL — very-low density lipoprotein.

pression during fasting is mediated by increased glucocorticoid levels and their binding to negative glucocorticoid responsive elements in the promotor region [24].

In summary, ANGPTL3, ANGPTL4 and ANGPTL8 together ensure that triglycerides from triglyceride-rich lipoproteins are properly distributed under various physiological conditions [22].

\section{ANGPTL3, 4 and 8 and cardiovascular risk: Observational studies}

In a study by Morinaga et al. [25] involving 988 Japanese, ANGPTL3, 4 and 8 serum concentration and cardiovascular risk were analyzed. ANGPTL serum concentration measurement by enzyme- -linked immunosorbent assay. Serum ANGPTL3 concentrations have been shown to be relatively high in patients with hepatic impairment and inflammation. Serum ANGPTL4 concentrations were also significantly increased in patients with impaired glucose metabolism, liver failure, but decreased in inflammation. In addition, an increase in serum ANGPTL8 concentration was observed in patients with glucose metabolism disorders, obesity and dyslipidemia. In particular, the increased ANGPTL8 serum concentration was positively correlated with serum triglyceride and LDL cholesterol concentration and inversely correlated with serum HDL cholesterol concentration. Therefore, it was found that the concentration of ANGPTL3, 4 and 8 in serum reflects the occurrence of some 


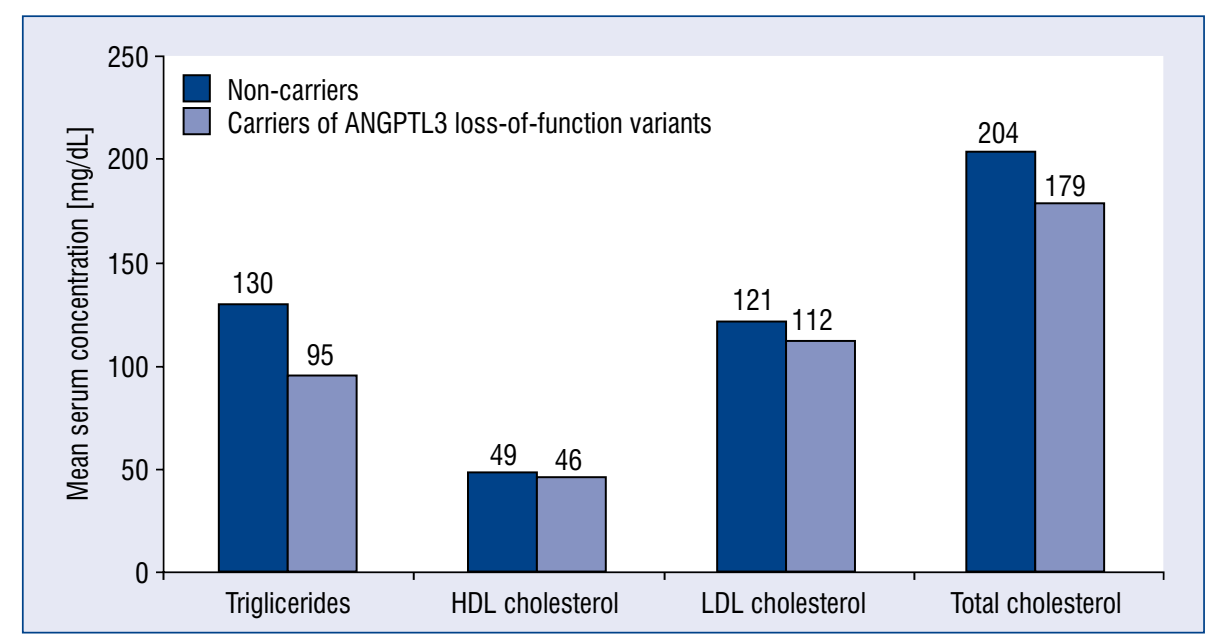

Figure 4. Associations between angiopoietin-like protein 3 (ANGPTL3) predicted loss-of-function variants and lipid serum concentrations in DiscovEHR Study participants. Based on [26]; HDL — high density lipoprotein; LDL — low density lipoprotein.

risk factors for the development of cardiovascular diseases [25]. In a study by Dewey et al. [26], the effect of ANGPTL3 gene polymorphism on triglycerides, LDL and HDL serum concentrations were analyzed. We sequenced the ANGPTL3 exons in 58.335 participants of the DiscovEHR human genetics study. Association tests for variants of loss of function in ANGPTL3 with lipid serum concentration and coronary artery disease (CAD) were carried out in 13.102 patient cases and 40.430 controls from the DiscovEHR study, with further studies involving 23.317 patient cases and 107.166 patients from four population studies. It was shown that people who were heterozygous in terms of loss of function in ANGPTL3 had significantly decreased triglycerides, HDL and LDL serum concentrations (Fig. 4) [26].

Moreover, it was found that people heterozygous for loss of ANGPTL3 gene function were characterized by a less incidence of cardiovascular disease (Fig. 5) [26].

Sitiziel et al. [27] assessed the effect of ANGPTL3 deficiency on CAD risk. Three subjects with total ANGPTL3 deficiency were included in the study. In the population mutations of loss of ANGPTL3 function (LOF) were found in up to 21.980 subjects with $\mathrm{CAD}$ and 158.200 control subjects. LOF mutations were defined as nonsense variants, frame shift and splice site, along with missense variants giving $<25 \%$ wild type ANGPTL3 activity in the mouse model. The biomarker study measured circulating ANGPTL3 in 1493 subjects with myocardial infraction and 3232 controls. It was shown that subjects with complete ANGPTL3 deficiency did not have coronary plaque. Sequencing of the ANGPTL3 genes showed that about 1 in 309 people were heterozygous carriers of the LOF mutation. Compared with those without mutations, heterozygous carriers of the ANGPTL3 LOF mutation showed a $17 \%$ reduction in circulating TG and a $12 \%$ reduction in LDL. The carrier's status was associated with a $34 \%$ decrease in the CAD chance. People with the lowest tertile in circulating ANGPTL3 concentrations, compared to the highest, had a reduced chance of myocardial infraction (Fig. 6) [27].

The results of these observational studies indicate that increased ANGPTL3, 4 and 8 may be associated with increased cardiovascular risk [25-27].

\section{ANGPTL inhibitors: Experimental and pre-clinical studies}

In 2002, Koishi et al. [28] identified an insertional mutation ANGPTL3 gene associated with hypolipidemic phenotype in obese knockout mice (KK/San mice). TG, total cholesterol and free fatty acids plasma concentrations in these mice were lower than in wild-type mice [28]. These observations were confirmed in the studies of Shimamura et al. [29, 30] in which wild-type KK mice showed signs of obesity accompanied by hyperinsulinemia and hypertriglyceridemia. The mutant strain of mice (KK/San and KK/Snk; mice expressing truncated ANGPTL3) were characterized by obesity 


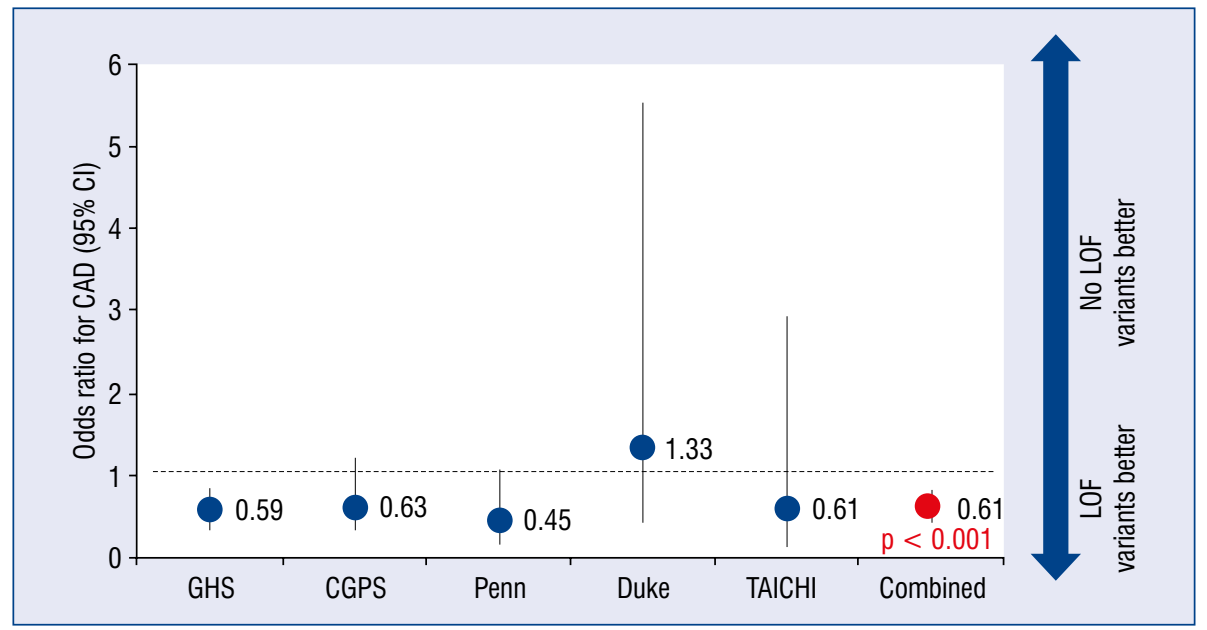

Figure 5. Association of angiopoietin-like protein 3 (ANGPTL3) loss of function (LOF) variants and coronary artery disease (CAD). Based on [26]; Cl — confidence interval; GHS - Geisinger Health System (DiscovEHR study); CGPS - Copenhagen General Population Studies; Penn — Penn the University of Pennsylvania Medicine BioBank; Duke Duke Catheterization Genetics cohort; TAICHI — Taiwan Metabochip consortium.

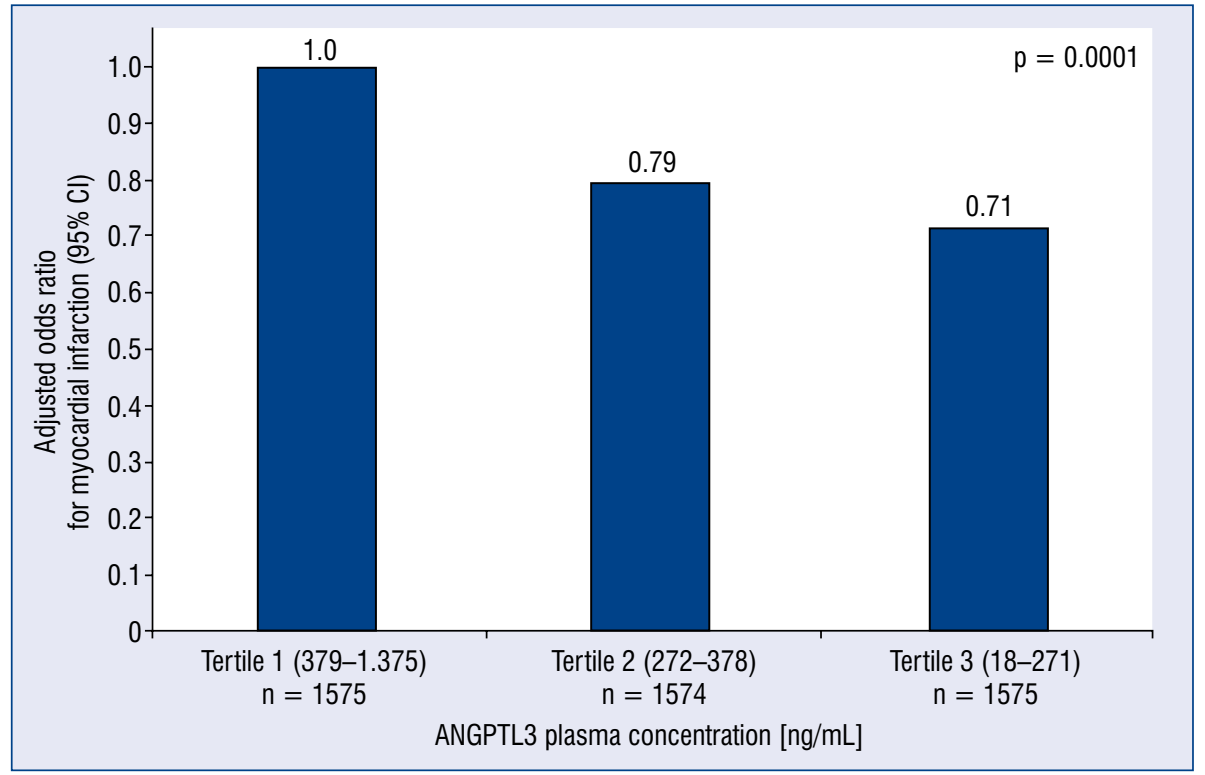

Figure 6. Association of angiopoietin-like protein 3 (ANGPTL3) plasma concentration with myocardial infarction risk. Based on [27]; $\mathrm{Cl}$ - confidence interval.

and diabetes with a $>90 \%$ lower TG plasma concentration compared to wild-type mice. Administration of adenovirus with the normal ANGPTL3 gene to mutated mice resulted in increased TG plasma concentrations. To explain the effect of ANGPTL3 on TG metabolism, overexpression of the ANGPTL3 gene was induced in mice. Increases in plasma total cholesterol, non-esterified fatty acids, as well as TG-rich lipoproteins concentrations have been observed. Subsequent studies affirmed that there was no significant difference between mutant and wild-type KK mice in the hepatic VLDL TG secretion rate, but in vitro analysis of recombinant protein revealed that ANGPTL3 directly inhibited LPL activity [29, 30]. It was shown that the removal of the ANGPTL3 gene in mice increased LPL activity by approximately $1.6 \times$ compared to wild-type mice [31]. The same experimental model showed a significant fall in the uptake of circulating VLDL-TG into white adipose tissue, rather 


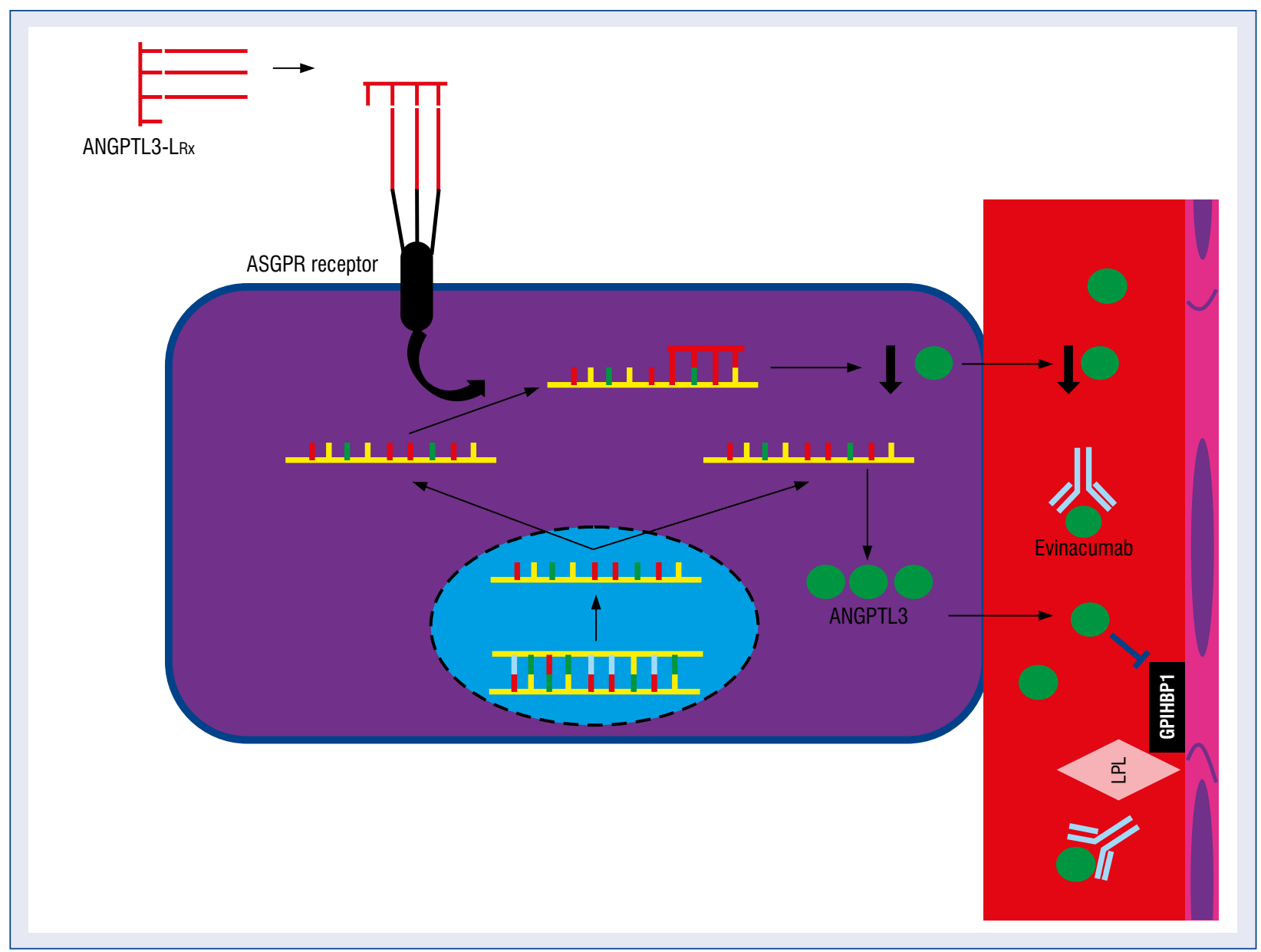

Figure 7. Mechanism of action of evinacumab and ANGPTL3- $L_{R x}$. Based on [34]; ANGPTL3 — angiopoietin-like protein 3; ANGPTL3- $L_{\mathrm{Rx}}$ — antisense oligonucleotide; ASGPR — asialoglycoprotein receptor. GPIHBP1 — glycosylphosphatidylinositol anchored high density lipoprotein binding protein 1; LPL — lipoprotein lipase.

than into skeletal muscle, brown adipose tissue and heart [32].

Thus, in experimental studies it was found that the lack of ANGPTL3 leads to a decrease plasma TG, total cholesterol, free fatty acids, VLDL, LDL and $\mathrm{apoB}_{100}$ concentrations [1].

Because genetic studies indicate that ANGPTL3 deficiency protects against atherosclerosis and that this is a causal relationship, work is ongoing on the anti-ANGPTL3 antibody (evinacumab).

Evinacumab (REGN1500) is a fully human monoclonal antibody (mAb) against ANGPTL3. This drug was developed by Regeneron Pharmaceuticals, and on April 6, 2017, the United States Food and Drug Administration (FDA) recognized evinacumab as a breakthrough therapy in the treatment of hypercholesterolemia in patients with homozygous familial hypercholesterolemia (HoFH). Evinacumab can be administered subcutaneously (sc) or intravenously $(i v)$. The formation of the evinacumab-ANGPTL3 complex reduces the biological activity of ANGPTL3 (Fig. 7) [33, 34].

In a study by Wang et al. [35] using evinacumab in mice with a genetic deficiency in key proteins involved in the clearance of $\mathrm{apoB}_{100}$ containing lipoproteins, it was shown that evinacumab treatment consistently reduced plasma total cholesterol concentrations in mice in which apoE, receptor LDL, Lrp1 and Sdc1 were inactivated alone or in combination. Despite a $61 \%$ reduction in VLDL-TG production, production of VLDL-apoB $\mathrm{B}_{100}$ did not change in evinacumab-treated animals. Hepatic TG content, fatty acid synthesis and fatty acid oxidation were similar in evinacumab and antibody treated control animals. In summary, researchers state that inactivation of ANGPTL3 does not affect the number of $\mathrm{apoB}_{100}$-containing lipoproteins secreted by the liver, but changes the produced particles in such a way that they are removed faster from the circulation [35]. Another study looked at the 
effect of administration of evinacumab that binds ANGPTL3 with high affinity to the lipid profile in normolipemic mice. Evinacumab reversed ANGPTL3-induced inhibition of LPL activity in vitro. Intravenously evinacumab administration of $\mathrm{C} 57 \mathrm{Bl} / 6$ normolipidemic mice increased LPL activity and decreased plasma TG concentrations by $\geq 50 \%$. In addition, chronic administration of evinacumab to $\mathrm{C} 57 \mathrm{Bl} / 6$ dyslipidemic mice for 8 weeks reduced plasma TG, LDL and HDL concentrations without any change in liver, fat or heart TG content. Studies in EL KK mice revealed that evinacumab reduced serum HDL concentration via an EL-dependent mechanism. Finally, administration of a single dose of evinacumab to dyslipidemic cynomolgus monkeys resulted in a rapid and pronounced decrease plasma TG, non-HDL and HDL concentration. Evinacumab normalized plasma TG concentration even in monkeys with baseline plasma TG concentration greater than $400 \mathrm{mg} / \mathrm{dL}$ [36]. Pouwer et al. [37] studied the effect of such lipid lowering interventions on atherosclerosis in APOE*3-Leiden. CETP mice, a well-established model of hyperlipidemia. The mice were fed a Western-type diet for 13 weeks and then matched to the basal group (died after 13 weeks) and five groups who received the diet alone (control) or with treatment (atorvastatin; atorvastatin and alirocum$\mathrm{ab}$; atorvastatin and evinacumab; or atorvastatin, alirocumab and evinacumab [triple therapy]) for 25 weeks. The impact of the intervention on cholesterol plasma concentration, plaque composition and morphology, monocyte adhesion and macrophage proliferation were analyzed. All interventions reduced total plasma cholesterol concentration (37\% with atorvastatin to $80 \%$ with triple treatment; all $\mathrm{p}<0.001)$. Triple treatment reduced non-HDL to $1.0 \mathrm{mmol} / \mathrm{L}$ (91\% difference compared to control; $\mathrm{p}<0.001$ ). Atorvastatin reduced the progression of atherosclerosis by $28 \%$ compared to control ( $\mathrm{p}<0.001)$; double treatment completely blocked the progression and reduced the severity of the lesions. Triple treatment reduced the size of the lesion compared to baseline in the thoracic aorta by $50 \%$ and aortic root by $36 \%$ (both $p<0.05$ compared to baseline), reduced macrophage accumulation through reduced proliferation, and reduced the severity of the lesion. Thus, triple cholesterol-lowering therapy, targeted at all $\mathrm{apoB}_{100}$-containing lipoproteins, regresses the area of atherosclerotic lesions and improves the composition of the lesions in mice [37]. In another interesting study, Graham et al. [38] evaluated the effect of the use of ANGPTL3 antisense oligonucleotides (ASO) on the lipid profile of mice with knockout of the LDL receptor $\left(\mathrm{LDL}^{-\mathrm{R}^{--}}\right.$), double-knockout mice (ApoC-III ${ }^{-/}$and LDL- ${ }^{-/-}$), heterozygous mice (ApoCIII ${ }^{+-}$and $\mathrm{LDL}^{-\mathrm{R}^{--}}$), mice with diet-induced obesity, and mice overexpressing human apolipoprotein C-III. The administration of murine ANGPTL3 ASO has been shown to lead to a decrease in ANGPTL3 mRNA expression from $69 \%$ to $91 \%$, which corresponds to a reduction in protein levels $50-90 \%$ in each of these mouse models. For lipid profile, TG, LDL and HDL were reduced, between 35-85\%, 7-64\% and $3-23 \%$, respectively. Importantly, ANGPTL3- $\mathrm{L}_{\mathrm{Rx}}$ also reduces liver TG secretion, which suggests that an ANGPTL3 targeted drug could be used to treat fatty liver [38]. Other studies on wild-type and hyperlipemic LDL- $\mathrm{R}^{-/-}$mice, in which the ANGPTL3 gene was edited using the CRISP-Cas9 (clustered regularly-interspaced short palindromic repeats) method, showed a decreased plasma TG and total cholesterol concentration by $31 \%$ and $19 \%$ and $56 \%$, and $51 \%$, respectively [39].

Interestingly, the use of anti-ANGPTL4 monoclonal antibody (anti-ANGPTL4 mAb) on high-fat diet fed mice led to decrease TG concentration by $50 \%$ and $59 \%$, and total cholesterol by $30 \%$. In the LDL- $\mathrm{R}^{--}$or $\mathrm{db} / \mathrm{db}$ group of mice, this intervention led to decrease plasma TG concentration by $55 \%$ and total cholesterol by $25 \%$. In the apoE $\mathrm{E}^{--}$mouse group, no changes in lipid profile were observed [40].

Less interest in ANGPTL4 as a therapeutic target is due to reported adverse effects such as mesenteric adenitis in rodents treated with anti-ANGPTL4 antibody [41].

\section{ANGPTL inhibitors: Clinical studies}

\section{Evinacumab}

The first phase double-blind, placebo-controlled clinical study evaluated the safety and efficacy of $s c$ or $i v$ evinacumab in patients with elevated $\mathrm{TG}(150) \leq \mathrm{TG} \leq 450 \mathrm{mg} / \mathrm{dL})$ and/or LDL $(\geq 100$ $\mathrm{mg} / \mathrm{dL}$ ). In this study randomized participants to placebo (9, PBOsc; $12, \mathrm{PBO} i v)$ and evinacumab (11, $75 \mathrm{mg} \mathrm{sc} ; 12,150 \mathrm{mg} \mathrm{sc} ; 9,250 \mathrm{mg} \mathrm{sc} ; 10,5 \mathrm{mg} / \mathrm{kg}$ $i v ; 9,10 \mathrm{mg} / \mathrm{kg} i v ; 11,20 \mathrm{mg} / \mathrm{kg} i v)$. Evinacumab was shown to be well tolerated in the study. The most common adverse reaction was headache (11.3\% in the evinacumab group). There was no dose related safety trend. Evinacumab caused a dose-dependent reduction plasma TG concentration by $1.0 \%$ to $75.0 \%$ and LDL by $3.4 \%$ to $25.5 \%$. A dose-dependent decrease plasma HDL, VLDL, total cholesterol, non-HDL cholesterol, $\mathrm{ApoA}_{1}$ and $\mathrm{ApoB}_{100}$ concentrations was also observed, but 
no apparent effect on $\mathrm{Lp}$ (a) [42]. Similar results were obtained in the study by Dewey et al. [26] 83 volunteers with mild to moderately elevated triglycerides ( 150 to $450 \mathrm{mg} / \mathrm{dL}$ ) or LDL cholesterol $(\geq 100 \mathrm{mg} / \mathrm{dL}$ ) were included in the study. It was shown that the maximum changes in lipid plasma concentrations found in patients who received the $20 \mathrm{mg} i v$ dose per kilogram were as follows: TG $-76.0 \%$ (day 4 ); directly measured LDL $23.2 \%$ (day 15); and HDL - 18.4\% (day 15) [26]. Ahmad et al. [43] evaluated the effect of administering evinacumab in patients with TG $>150$ but $\leq 450 \mathrm{mg} / \mathrm{dL}$ and LDL cholesterol $\geq 100 \mathrm{mg} / \mathrm{dL}$ $(\mathrm{n}=83$ in a single increasing dose study [SAD]; $\mathrm{n}=56$ in the multiple dose escalation study [MAD]), they were randomized 3:1 to evinacumab: placebo. SAD patients received evinacumab $s c$ at a dose of 75/150/250 mg or $i v$ at a dose of 5/10/20 $\mathrm{mg} / \mathrm{kg}$, monitored up to 126 days. MAD patients received evinacumab $s c$ in doses of 150/300/450 mg once a week, 300/450 mg every 2 weeks or $i v$ at a dose of $20 \mathrm{mg} / \mathrm{kg}$ once every 4 weeks to 56 days after 6 months of observation. There was a dose-dependent decrease in TG plasma concentration, with a maximum reduction of $76.9 \%$ on day 3 at $10 \mathrm{mg} / \mathrm{kg} i v(\mathrm{p}<0.0001)$ in SAD and $83.1 \%$ on day 2 when intravenous $20 \mathrm{mg} / \mathrm{kg}$ once daily 4 weeks $(p=0.0003)$ in MAD. Significant reduction of other lipids was observed at most doses of evinacumab compared to placebo [43]. Gaudet et al. [44] tested evinacumab in a group of 9 adults with familial hypercholesterolemia. Patients received evinacumab at a dose of $250 \mathrm{mg} \mathrm{sc}$ at the beginning and $15 \mathrm{mg} / \mathrm{kg} i v$ in the second week of the study. After 4 weeks of treatment, evinacumab reduced LDL plasma concentration by an average of $49 \pm$ $\pm 23 \%$ (range $25-90 \%$ ), with an absolute decrease from baseline $157 \pm 90 \mathrm{mg} / \mathrm{dL}$ (range 71-323). In addition, a significant $-48 \%$ - decrease in apoB ${ }_{100}$, non-HDL and TG plasma concentrations has been demonstrated [44]. ANGPTL3 inhibitors are characterized by good efficacy in the treatment of patients with HoFH. Hovingh et al. [45] in a study of 9 patients with HoFH evaluated the effect of evinacumab on LDL plasma concentrations. Evinacumab was dosed as a single $250 \mathrm{mg} s c$ injection at baseline and subsequently as $15 \mathrm{mg} / \mathrm{kg} \mathrm{iv}$ at week 2. Two patients further received $450 \mathrm{mg}$ $s c$ at weeks $12,13,14$, and 15 . The primary endpoint was the mean percent change in LDL plasma concentrations from baseline to week 4 . The mean baseline LDL plasma concentration was $376 \mathrm{mg} / \mathrm{dL}$. After 4 weeks of evinacumab therapy, LDL plasma concentrations decreased by an average of $49.2 \%$.
The maximum reduction in LDL plasma concentration ranged from $33 \%$ to $90 \%$. It has been found that these preliminary results give great hope that inhibition of ANGPTL3 by evinacumab is likely to result in a clinically significant reduction in LDL plasma concentrations in patients with $\mathrm{HoFH}$ [45]. In another study by Banerjee et al. [46] which evaluated LDL receptor activity in $\mathrm{HoFH}$ patients' lymphocytes before and after treatment with evinacumab in comparison with the wild-type LDL receptor-positive lymphocytes, as well as in the LDL receptor defective Chinese hamster ovary cell line (CHO-LDLA7) transfected with plasmids encoding the LDL variant receptor. The overall mean maximum reduction in plasma LDL concentration with evinacumab was $-58 \pm 18 \%$, occurring between weeks 4 and 12 . The mutations identified in 9 patients were shown to be pathogenic, with a loss of LDL receptor activity as compared to the wildtype. Two of the LDL receptor variants (Cys681* and Ala627Profs*38, were type 2 mutations that are retained in the endoplasmic reticulum. Six variants are class 3 mutations with impaired LDL binding activity (Trp87Gly), occurred in 2 patients (Gln254Pro; Ser177Leu; Gly335Val; Ser306Leu). Evinacumab had no effect on LDL receptor activity. Researchers found that evinacumab is effective in lowering plasma LDL in $\mathrm{HoFH}$ patients, and inhibiting ANGPTL3 in humans reduces plasma LDL in a mechanism independent of LDL receptor [46].

Raal [47] during the American College of Cardiology Congress in 2020 presented the results of a study involving 65 patients, 12 years of age or older with $\mathrm{HoFH}$ and plasma LDL concentrations greater than $70 \mathrm{mg} / \mathrm{dL}$ at screening. After an 8 -week lead-in period to stabilize basic lipid-lowering therapy, 43 patients were randomized to receive evinacumab $15 \mathrm{mg} / \mathrm{kg} i v$ every 4 weeks and 22 to placebo group. Following a 24 -week double-blind treatment period, there was a 24 -week extension to the open label period during which all patients received the study drug. More than two-thirds of patients had some residual LDL receptor function - non-null/null genotype status - while about $30 \%$ had minimal or no LDL receptor function null/null genotype status. Over $90 \%$ of patients were taking a statin, over two-thirds were taking ezetimibe, about $20 \%$ were taking lomitapib, and just over one-third were taking apheresis. Despite these treatments, mean plasma concentrations of LDL and apoB $B_{100}$ in patients on entry were about $250 \mathrm{mg} / \mathrm{dL}$ and about $170 \mathrm{mg} / \mathrm{dL}$, respectively. Evinacumab has been shown to significantly reduce plasma LDL (by $47.1 \%$ ), apoB ${ }_{100}$ by $36.9 \%$, non- 
Table 1. Outcomes in patients with familial hypercholesterolemia. Based on [47].

\begin{tabular}{lcccc}
\hline & Evinacumab & Placebo & Difference & P \\
\hline Percent change LDL plasma concentration & $-47.1 \%$ & $1.9 \%$ & $-49.0 \%$ & $<0.0001$ \\
Absolute change in LDL plasma concentration $[\mathrm{mg} / \mathrm{dL}]$ & -134.7 & -2.6 & -132.1 & $<0.0001$ \\
Patients with $\geq 30 \%$ reduction in LDL plasma concentration $[\%]$ & $83.7 \%$ & $18.2 \%$ & - & $<0.0001$ \\
Patients with $\geq 50 \%$ reduction in LDL plasma concentration $[\%]$ & $55.8 \%$ & $4.5 \%$ & - & 0.003 \\
Patients with LDL plasma concentration $<100 \mathrm{mg} / \mathrm{dL}$ & $46.5 \%$ & $22.7 \%$ & - & 0.02 \\
\hline
\end{tabular}

LDL — low density lipoprotein

HDL cholesterol by $51.7 \%$, total cholesterol by $48.4 \%$, and triglycerides by $50.4 \%$ ( $\mathrm{p}<0.0001$ for all) concentrations. However, the study drug had no effect on Lp(a) plasma concentration (Table 1) [47].

Currently, six clinical trials using evinacumab are underway in: healthy volunteers (NCT03146416), patients with persistent hypercholesterolemia (NCT03175367), patients with HoHF (NCT03399786 and NCT03409744), children with HoHF (NCT04233918) and patients with severe hypertriglyceridemia and high risk of acute pancreatitis (NCT03452228).

\section{ANGPTL3-L $\mathrm{Lx}_{\mathrm{Rx}}$}

ANGPTL3- $\mathrm{L}_{\mathrm{Rx}}$ are antisense oligonucleotides containing three GalNax residues which promote the specific recognition by hepatic ASGPR receptors (asialoglycoprotein receptor). Once internalized, ANGPTL3- $\mathrm{L}_{\mathrm{Rx}}$ inhibit protein synthesis of ANGPTL3, respectively, by binding the corresponding mRNAs and inducing their degradation (Fig. 7) [34].

The use of ANGPTL3- $\mathrm{L}_{\mathrm{Rx}}$ in healthy subjects aged 18-65 years with TG $>150 \mathrm{mg} / \mathrm{dL}$ (at doses of $10,20,40$ and $60 \mathrm{mg} /$ week for 6 weeks) resulted in a decrease in plasma $\mathrm{TG}$ concentration from $33.2 \%$ to $63 \%$, LDL by $1.3 \%$ to $32.9 \%$ and VLDL by $27.9 \%$ to $60 \%$ [37]. No side effects or serious adverse effects were noted, but clinical data are yet very limited [37].

There is currently insufficient information on the long-term safety of evinacumab and ANGPTL3- $\mathrm{L}_{\mathrm{Rx}}$. As both drugs are still in the early stages of development, it has not yet been proven that the reduction in plasma LDL concentrations achieved with evinacumab and ANGPTL3- $\mathrm{L}_{\mathrm{Rx}}$ will reduce cardiovascular risk, until there is clinical experience [33].

\section{Conclusions}

1. ANGPTL3, ANGPTL4 and ANGPTL8 play an important role in regulating lipoprotein metabolism. These proteins are involved in the regulation of triglyceride availability depending on the nutritional status of the body.

2. Observational studies have shown that increased plasma ANGPTL3, 4 and 8 concentrations may reflect cardiovascular risk.

3. Observational studies have shown that low plasma concentrations of ANGPTL3, 4 and 8 appear to reduce the risk of cardiovascular disease.

4. Animal studies have shown that reducing ANGPTL3 activity leads to a decrease in plasma triglycerides, total cholesterol, VLDL, LDL and HDL concentrations.

5. The results of clinical studies indicate that the use of evinacumab and ANGPTL3- $\mathrm{L}_{\mathrm{Rx}}$ is effective and safe in the treatment of patients with dyslipidemia and $\mathrm{HoHF}$.

6. The lipid-lowering effect of evinacumab is independent of the type of LDL receptor mutation.

Conflict of interest: None declared

\section{References}

1. Ruscica M, Zimetti F, Adorni MP, et al. Pharmacological aspects of ANGPTL3 and ANGPTL4 inhibitors: New therapeutic approaches for the treatment of atherogenic dyslipidemia. Pharmacol Res. 2020; 153: 104653, doi: 10.1016/j.phrs.2020.104653, indexed in Pubmed: 31931117.

2. Santulli G. Angiopoietin-like proteins: a comprehensive look. Front Endocrinol (Lausanne). 2014; 5: 4, doi: 10.3389/fendo.2014.00004, indexed in Pubmed: 24478758.

3. Tarugi P, Bertolini S, Calandra S. Angiopoietin-like protein 3 (ANGPTL3) deficiency and familial combined hypolipidemia. J Biomed Res. 2019; 33(2): 73-81, doi: 10.7555/JBR.32.20170114, indexed in Pubmed: 29752428.

4. Hato T, Tabata M, Oike Y. The role of angiopoietin-like proteins in angiogenesis and metabolism. Trends Cardiovasc Med. 2008; 18(1): 6-14, doi: 10.1016/j.tcm.2007.10.003, indexed in Pubmed: 18206803.

5. Lupo MG, Ferri N. Angiopoietin-lke 3 (ANGPTL3) and atherosclerosis: lipid and non-lipid related effects. J Cardiovasc Dev 
Dis. 2018; 5(3), doi: 10.3390/jcdd5030039, indexed in Pubmed: 30011918.

6. Essalmani R, Susan-Resiga D, Chamberland A, et al. Furin is the primary in vivo convertase of angiopoietin-like 3 and endothelial lipase in hepatocytes. J Biol Chem. 2013; 288(37): 26410-26418, doi: 10.1074/jbc.M113.501304, indexed in Pubmed: 23918928.

7. Quagliarini F, Wang Y, Kozlitina J, et al. Atypical angiopoietin-like protein that regulates ANGPTL3. Proc Natl Acad Sci USA. 2012; 109(48): 19751-19756, doi: 10.1073/pnas.1217552109, indexed in Pubmed: 23150577.

8. Haller JF, Mintah IJ, Shihanian LM, et al. ANGPTL8 requires ANGPTL3 to inhibit lipoprotein lipase and plasma triglyceride clearance. J Lipid Res. 2017; 58(6): 1166-1173, doi: 10.1194/jlr. M075689, indexed in Pubmed: 28413163.

9. Kaplan R, Zhang T, Hernandez M, et al. Regulation of the angiopoietin-like protein 3 gene by LXR. J Lipid Res. 2003; 44(1): 136-143, doi: 10.1194/jlr.m200367-jlr200, indexed in Pubmed: 12518032 .

10. Foka P, Karamichali E, Dalagiorgou G, et al. Hepatitis C virus modulates lipid regulatory factor Angiopoietin-like 3 gene expression by repressing HNF-1 $\alpha$ activity. J Hepatol. 2014; 60(1): 30-38, doi: 10.1016/j.jhep.2013.08.016, indexed in Pubmed: 23978712 .

11. Ortega-Senovilla H, Schaefer-Graf U, Meitzner K, et al. Decreased concentrations of the lipoprotein lipase inhibitor angiopoietin-like protein 4 and increased serum triacylglycerol are associated with increased neonatal fat mass in pregnant women with gestational diabetes mellitus. J Clin Endocrinol Metab. 2013; 98(8): 3430-3437, doi: 10.1210/jc.2013-1614, indexed in Pubmed: 23744407.

12. Nakajima K, Tokita Y, Tanaka A, et al. The VLDL receptor plays a key role in the metabolism of postprandial remnant lipoproteins. Clin Chim Acta. 2019; 495: 382-393, doi: 10.1016/j. cca.2019.05.004, indexed in Pubmed: 31078566.

13. Olivecrona G. Role of lipoprotein lipase in lipid metabolism. Curr Opin Lipidol. 2016; 27(3): 233-241, doi: 10.1097/ MOL.0000000000000297, indexed in Pubmed: 27031275.

14. Shimizugawa T, Ono M, Shimamura M, et al. ANGPTL3 decreases very low density lipoprotein triglyceride clearance by inhibition of lipoprotein lipase. J Biol Chem. 2002; 277(37): 33742-33748, doi: 10.1074/jbc.M203215200, indexed in Pubmed: 12097324.

15. Yu JE, Han SY, Wolfson B, et al. The role of endothelial lipase in lipid metabolism, inflammation, and cancer. Histol Histopathol. 2018; 33(1): 1-10, doi: 10.14670/HH-11-905, indexed in Pubmed: 28540715 .

16. Liu J, Afroza H, Rader DJ, et al. Angiopoietin-like protein 3 inhibits lipoprotein lipase activity through enhancing its cleavage by proprotein convertases. J Biol Chem. 2010; 285(36): 27561-27570, doi: 10.1074/jbc.M110.144279, indexed in Pubmed: 20581395.

17. Mattijssen F, Kersten S. Regulation of triglyceride metabolism by Angiopoietin-like proteins. Biochim Biophys Acta. 2012; 1821(5): 782-789, doi: 10.1016/j.bbalip.2011.10.010, indexed in Pubmed: 22063269 .

18. Chi X, Shetty SK, Shows HW, et al. Angiopoietin-like 4 modifies the interactions between lipoprotein lipase and its endothelial cell transporter GPIHBP1. J Biol Chem. 2015; 290(19): 11865-11877, doi: 10.1074/jbc.M114.623769, indexed in Pubmed: 25809481.

19. Haller JF, Mintah IJ, Shihanian LM, et al. ANGPTL8 requires ANGPTL3 to inhibit lipoprotein lipase and plasma triglyceride clearance. J Lipid Res. 2017; 58(6): 1166-1173, doi: 10.1194/jlr. M075689, indexed in Pubmed: 28413163.

20. Kovrov O, Kristensen KK, Larsson E, et al. On the mechanism of angiopoietin-like protein 8 for control of lipoprotein lipase activity. J Lipid Res. 2019; 60(4): 783-793, doi: 10.1194/jlr.M088807, indexed in Pubmed: 30686789.

21. Mead JR, Irvine SA, Ramji DP. Lipoprotein lipase: structure, function, regulation, and role in disease. J Mol Med (Berl). 2002; 80(12): 753-769, doi: 10.1007/s00109-002-0384-9, indexed in Pubmed: 12483461.

22. Dijk W, Kersten S. Regulation of lipid metabolism by angiopoietin-like proteins. Curr Opin Lipidol. 2016; 27(3): 249-256, doi: 10.1097/MOL.0000000000000290, indexed in Pubmed: 27023631.

23. Aryal B, Price NL, Suarez Y, et al. ANGPTL4 in metabolic and cardiovascular disease. Trends Mol Med. 2019; 25(8): 723-734, doi: 10.1016/j.molmed.2019.05.010, indexed in Pubmed: 31235370 .

24. Dang F, Wu R, Wang P, et al. Fasting and feeding signals control the oscillatory expression of ANGPTL8 to modulate lipid metabolism. Sci Rep. 2016; 6: 36926, doi: 10.1038/srep36926, indexed in Pubmed: 27845381.

25. Morinaga J, Zhao J, Endo M, et al. Association of circulating ANGPTL 3, 4, and 8 levels with medical status in a population undergoing routine medical checkups: a cross-sectional study. PLoS One. 2018; 13(3): e0193731, doi: 10.1371/journal. pone.0193731, indexed in Pubmed: 29538435.

26. Dewey FE, Gusarova V, Dunbar RL, et al. Genetic and pharmacologic inactivation of ANGPTL3 and cardiovascular disease. N Engl J Med. 2017; 377(3): 211-221, doi: 10.1056/NEJMoa1612790, indexed in Pubmed: 28538136.

27. Stitziel NO, Khera AV, Wang X, et al. ANGPTL3 deficiency and protection against coronary artery disease. J Am Coll Cardiol. 2017; 69(16): 2054-2063, doi: 10.1016/j.jacc.2017.02.030, indexed in Pubmed: 28385496.

28. Koishi R, Ando Y, Ono M, et al. ANGPTL3 regulates lipid metabolism in mice. Nat Genet. 2002; 30(2): 151-157, doi: 10.1038/ ng814, indexed in Pubmed: 11788823.

29. Shimamura M, Matsuda M, Kobayashi S, et al. Angiopoietin-like protein 3, a hepatic secretory factor, activates lipolysis in adipocytes. Biochem Biophys Res Commun. 2003; 301(2): 604-609, doi: 10.1016/s0006-291x(02)03058-9.

30. Shimamura M, Matsuda M, Yasumo H, et al. Angiopoietin-like protein3 regulates plasma HDL cholesterol through suppression of endothelial lipase. Arterioscler Thromb Vasc Biol. 2007; 27(2): 366-372, doi: 10.1161/01.ATV.0000252827.51626.89, indexed in Pubmed: 17110602 .

31. Fujimoto K, Koishi R, Shimizugawa T, et al. Angptl3-null mice show low plasma lipid concentrations by enhanced lipoprotein lipase activity. Exp Anim. 2006; 55(1): 27-34, doi: 10.1538/expanim.55.27, indexed in Pubmed: 16508209.

32. Wang Y, McNutt MC, Banfi S, et al. Hepatic ANGPTL3 regulates adipose tissue energy homeostasis. Proc Natl Acad Sci USA. 2015; 112(37): 11630-11635, doi: 10.1073/pnas.1515374112, indexed in Pubmed: 26305978.

33. Strilchuk L, Fogacci F, Cicero AFg. Safety and tolerability of injectable lipid-lowering drugs: an update of clinical data. Expert Opin Drug Saf. 2019; 18(7): 611-621, doi: 10.1080/14740338.2019.1620730, indexed in Pubmed: 31100030.

34. Pirillo A, Norata GD, Catapano AL. LDL-cholesterol-lowering therapy. Handb Exp Pharmacol. Springer, Berlin, Heidelberg. 
2020 [Epub ahead of print], doi: 10.1007/164_2020_361, indexed in Pubmed: 32350699.

35. Wang Y, Gusarova V, Banfi S, et al. Inactivation of ANGPTL3 reduces hepatic VLDL-triglyceride secretion. J Lipid Res. 2015; 56(7): 1296-1307, doi: 10.1194/jlr.M054882, indexed in Pubmed: 25954050.

36. Gusarova V, Alexa CA, Wang Y, et al. ANGPTL3 blockade with a human monoclonal antibody reduces plasma lipids in dyslipidemic mice and monkeys. J Lipid Res. 2015; 56(7): 1308-1317, doi: 10.1194/jlr.M054890, indexed in Pubmed: 25964512.

37. Pouwer MG, Pieterman EJ, Worms N, et al. Alirocumab, evinacumab, and atorvastatin triple therapy regresses plaque lesions and improves lesion composition in mice. J Lipid Res. 2020; 61(3): 365-375, doi: 10.1194/j1r.RA119000419, indexed in Pubmed: 31843957.

38. Graham MJ, Lee RG, Brandt TA, et al. Cardiovascular and Metabolic Effects of ANGPTL3 Antisense Oligonucleotides. N Engl J Med. 2017; 377(3): 222-232, doi: 10.1056/NEJMoa1701329, indexed in Pubmed: 28538111.

39. Chadwick AC, Evitt NH, Lv W, et al. Reduced blood lipid levels with in vivo CRISPR-Cas9 base editing of ANGPTL3. Circulation. 2018; 137(9): 975-977, doi: 10.1161/CIRCULATIONAHA.117.031335, indexed in Pubmed: 29483174.

40. Desai U, Lee EC, Chung K, et al. Lipid-lowering effects of anti-angiopoietin-like 4 antibody recapitulate the lipid phenotype found in angiopoietin-like 4 knockout mice. Proc Natl Acad Sci USA. 2007; 104(28): 11766-11771, doi: 10.1073/pnas.0705041104, indexed in Pubmed: 17609370.

41. Dewey FE, Gusarova V, O'Dushlaine $\mathrm{C}$, et al. Inactivating variants in ANGPTL4 and risk of coronary artery disease. N Engl
J Med. 2016; 374(12): 1123-1133, doi: 10.1056/NEJMoa1510926, indexed in Pubmed: 26933753.

42. Dunbar R, Bouzelmat A, Pordy G, et al. Abstract 19133: Inhibition of ANGPTL 3 by Evinacumab Reduced Triglycerides (TGs) and LDL-C in Subjects Presenting With Modest Elevations in TGs and/or LDL-C, Recapitulating the Hypolipidemic Effects of Loss-of-Function (LoF) Mutations of ANGPTL3. Circulation. 2016; 134: A19133.

43. Ahmad Z, Banerjee P, Hamon S, et al. Inhibition of angiopoietinlike protein 3 with a monoclonal antibody reduces triglycerides in hypertriglyceridemia. Circulation. 2019; 140(6): 470-486, doi: 10.1161/CIRCULATIONAHA.118.039107, indexed in Pubmed: 31242752 .

44. Gaudet D, Gipe DA, Pordy R, et al. ANGPTL3 inhibition in homozygous familial hypercholesterolemia. N Engl J Med. 2017; 377(3): 296-297, doi: 10.1056/NEJMc1705994, indexed in Pubmed: 28723334.

45. Hovingh GK, Gipe DA, Ahmad Z, et al. Efficacy of evinacumab in homozygous familial hypercholesterolemia patients with null or non-null LDL-receptor mutations and on various background therapies. Abstract 5938. Eur Heart J. 2017; 38(suppl_1), doi: 10.1093/eurheartj/ehx493.5938.

46. Banerjee P, Chan KC, Tarabocchia M, et al. Functional analysis of LDLR (low-density lipoprotein receptor) variants in patient lymphocytes to assess the effect of evinacumab in homozygous familial hypercholesterolemia patients with a spectrum of LDLR activity. Arterioscler Thromb Vasc Biol. 2019; 39(11): 22482260, doi: 10.1161/ATVBAHA.119.313051, indexed in Pubmed: 31578082.

47. Raal F. Evinacumab in patients with homozygous familial hypercholesterolemia. Presented on: March 30. 2020: ACC 2020. 